\title{
Polymerization of 1,4-dinitrosobenzene: Kinetics and Submicrocrystal Structure
}

\author{
Petar Bibulić, ${ }^{1}$ Igor Rončević, ${ }^{1, *}$ Vladimir Bermanec, ${ }^{2}$ Hrvoj Vančik ${ }^{1, \#}$
}

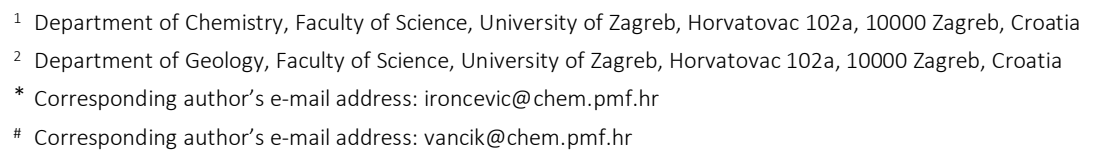

RECEIVED: September 19, 2017 * REVISED: October 10, 2017 * ACCEPTED: November 20, 2017

PROCEEDING OF THE 29 ${ }^{\mathrm{TH}}$ MATH /CHEM/COMP CONFERENCE, JUNE 19-25, 2017, DUBROVNIK, CROATIA THIS PAPER IS DEDICATED TO PROFESSOR IVAN GUTMAN ON THE OCCASION OF HIS $70^{\mathrm{TH}}$ BIRTHDAY

\begin{abstract}
In this study, the structure of the polycrystalline poly(l,4-phenyleneazine N,N-dioxide) was investigated by Scanning Electron Microscopy and related with the previously calculated molecular structure. Kinetics of polymerization of 1,4-dinitrosobenzene, prepared by both cryogenic UV photolysis and in vacuo deposition were measured by time-resolved FT-IR spectroscopy. Acquired data was analyzed by curve fitting with the standard Avrami-Erofeev and two-step consecutive reactions models. Activation parameters were calculated from Arrhenius and Eyring-Polanyi equations, for both models. The results obtained by using the two models, along with the goodness-of-fit parameters, were compared. It was shown that both bulk-based and reaction-based models can be used to adequately describe solid-state chemical reaction kinetics. Furthermore, a two-step consecutive reactions model is a suitable alternative to the most commonly used AvramiErofeev model.
\end{abstract}

Keywords: scanning electron microscopy, polymerization, kinetic models, solid state, activation parameters.

\section{INTRODUCTION}

A ROMATIC C-nitroso compounds have proven to be a useful model system for the investigation of chemical reactions in the solid state. ${ }^{[1-3]}$ They exist in two different forms, as nitroso compounds (monomers), and E- or Zazodioxy compounds (dimers or polymers). ${ }^{[4,5]}$ In the solid state, most aromatic C-nitroso compounds appear in the azodioxy form, but can be converted to stable or metastable monomeric forms by dissolution in organic solvents, heating, or by UV irradiation at cryogenic temperatures. ${ }^{[5]}$ An important feature of the nitroso/azodioxy system is that the two forms can be easily distinguished by spectroscopic methods (UV / Vis, NMR, IR, etc.), which facilitates kinetic measurements. [4]

1,4-Dinitrosobenzene has been investigated because it is, next to 1,3-dinitrosobenzene, the simplest aromatic Cdinitroso compound capable of polymerization. It has been found that by deposition of 1,4-dinitrosobenzene vapor onto the surface cooled to $12 \mathrm{~K}$ produces a solid layer consisting only of the nitroso monomer. ${ }^{[6,7]}$ Warming the sample to around $150 \mathrm{~K}$ causes fast polymerization. Recently, it has been found that the formation of polymers under cryogenic condition is not a simple process. The reaction on the cold surface follows the multistep mechanism. The first intermediate observed by IR spectroscopy is the Z-azodioxy isomer, but further warming causes the irreversible rearrangement to the $E$-isomer, and finally the formation of the stable polymer. Besides the use of cold surface deposition, 1,4-dinitrosobenzene can also be prepared photolytically. UV irradiation of the polymer encapsulated in a $\mathrm{KBr}$ pellet at $13 \mathrm{~K}$ yields monomers. However, these monomers dimerize and oligomerize exclusively to the azodioxy products with $E$-stereochemistry.

The polymerization mechanism as well as the stability of the polymer depends on the topotactic solid-state conditions. ${ }^{[3]}$ It appears that monomers are more randomly distributed after cryogenic deposition, while after photolysis of the polymer the orientation of monomer molecules is more conserved, which is convenient for 
repolymerization. ${ }^{[1,2]}$ Also, repolymerization after photolysis depends on the regularity of the crystal structure of the starting polymer. ${ }^{[1]}$ In this work, we present a morphological investigation of the polymeric material, complemented with isothermal determination of activation energies for the polymerization under cryogenic conditions.

For the analysis of isothermal polymerization, the standard Avrami-Erofeev (AE) approach is used along with the two-step consecutive reaction model (2CR). The reactions in the $2 \mathrm{CR}$ model can be written as:

$$
\mathrm{R} \stackrel{k_{1}}{\longrightarrow} \mathrm{R}^{*} \stackrel{\mathrm{k}_{2}}{\longrightarrow} \mathrm{P}
$$

where $R, R^{*}$ and $P$ are the reactant, the activated reactant and the product, respectively. The activated reactant $R^{*}$ can be thought of as a transient intermediate having a property (for example, spatial arrangement) which makes the reaction facile. In phase transitions, the activated reactants can be thought of as Avrami's germ nuclei, which might or might not form into active growth nuclei.[8-10] The motivation for using $2 \mathrm{CR}$ is that it is a reaction-order based model, meaning that it can give additional mechanistic insights about the underlying processes, while the $A E$ model only yields information about the overall transformation. More generally, the question is: Can reaction-order based models be used for the description of solid-state chemical reactions?

\section{EXPERIMENTAL}

\section{Synthesis}

Poly(l,4-phenyleneazine N,N-dioxide) (PNND) was prepared from cyclohexadiene-1,4-dione as previously described by Khishchenko.[11] The product was purified by vacuum sublimation, followed by condensation on a cold surface.

\section{Microscopy}

Zeiss electron microscope Gemini Supra installed at Laboratory for Microscopy and Microanalysis, University of Pretoria, RSA was used for obtaining high resolution SEM images. In Lens detector was employed at $1.7 \mathrm{~mm}$. Due to very sensitive material, working voltage was set just to $600 \mathrm{~V}$.

\section{Kinetic Measurements}

Samples for kinetic measurements were prepared either by photolysis of standard PNND doped KBr pellets ( $m$ (PNND) / $m(\mathrm{KBr}) \approx 1 / 100$ ) or by cryogenic deposition of 1,4-dinitrosobenzene vapors on a cooled $\mathrm{KBr}$ window. $\mathrm{KBr}$ pellets were secured on the cryocooler cold finger between two $\mathrm{KBr}$ discs and irradiated with $254 \mathrm{~nm}$ UV low-pressure Hg lamp (UVP R-52G). Samples were irradiated until no further changes in the IR spectra were noticeable.
Cryogenic conditions were achieved by LeyboldHeraeus ROK 10-300 cyclic helium cryostat. Isothermal kinetic measurements were performed at desired temperature after warming the samples from $13 \mathrm{~K}$ and holding the temperature constant. Temperature was control by Scientific Instruments model 9700 connected to a Si diode and a heater unit. Multiple measurements at different temperatures were conducted for the same sample to obtain activation parameters of repolymerization. FT-IR spectra were recorded on a PerkinElmer SpectrumTwo spectrometer, averaging 10 scans per spectrum. The background spectrum was obtained by observing ambient air. Spectra were recorded every $\sim 65 \mathrm{~s}$ and the reactions were monitored over a $2-4 \mathrm{~h}$ period. Solid-state repolymerization rates were measured following the change in absorbance of the $-\mathrm{N}=\mathrm{O}$ stretching at $1521 \mathrm{~cm}^{-1}, E-O N N O$ stretching at 1264 $\mathrm{cm}^{-1}$, or Z-ONNO stretching at $1387 \mathrm{~cm}^{-1}$. Baseline corrections for the spectra were performed using adaptive iteratively reweighted penalized least squares algorithm. Amount of the compound of interest was calculated according to the following equation:

$$
x_{Y}^{\prime}(t)=\frac{A_{Y}(t)-A_{Y}\left(t_{\text {end }}\right)}{A_{Y}\left(t_{\text {end }}\right)-A_{Y}\left(t_{\text {start }}\right)}
$$

where $x_{Y}^{\prime}(t)$ is the normalized amount of compound $Y$ present in time $t, A_{Y}\left(t_{\text {start }}\right)$ and $A_{Y}\left(t_{\text {end }}\right)$ are absorbances at the start and end of the measurement, respectively. Therefore, the normalized amount of the product is 0 at $t_{\text {start }}$ and 1 at $t_{\text {end. }}$.

\section{Avrami-Erofeev Model}

The description solid-state kinetics has proven to be a difficult problem for which a variety of solutions has been devised, with the AE model being the most prevalent. ${ }^{[12,13]}$ An often used form of the AE model is:

$$
x_{p}^{\prime}(t)=x_{\infty}^{\prime}-\left(x_{\infty}^{\prime}-x_{0}^{\prime}\right) \exp \left[-(k t)^{m}\right]
$$

Here, $x_{\infty}^{\prime}$ and $x_{0}^{\prime}$ are used as fitting parameters, along with the rate constant $k$ and the dimensionality coefficient $m$. This implementation of the Avrami equation often gives better variances than the standard implementation:

$$
\alpha(t)=1-e^{-(k t)^{n}}
$$

despite having a larger number of parameters. ${ }^{[6]}$ The $A E$ model has been successfully used to describe many solidstate processes-phase transitions, nanocluster formation, crystallization and chemical reactions. ${ }^{[13-16]}$ 


\section{Two-Step Consecutive Reaction Model}

From [Eq. (1)], the following differential equations can be written:

$$
\begin{aligned}
& \frac{d x_{R}^{\prime}}{d t}=k_{1} x_{R}^{\prime} \\
& \frac{d x_{R^{*}}^{\prime}}{d t}=k_{1} x_{R}^{\prime}-k_{2} x_{R^{*}}^{\prime} \\
& \frac{d x_{P}^{\prime}}{d t}=k_{2} x_{R^{*}}^{\prime}
\end{aligned}
$$

[Eq. (5)]-[Eq. (7)] can be analytically solved. If, $x_{R_{0}}^{\prime}$ $x_{R^{*}}^{\prime}$, and $x_{P_{0}}^{\prime}$ are the starting amounts of the reactant, activated reactant and product, the following equation can be used to calculate the amount of the product at time $t$ :

$$
x_{p}^{\prime}(t)=x_{R_{0}}^{\prime}\left(1+\frac{k_{1} e^{-k_{2} t}-k_{2} e^{-k_{1} t}}{k_{2}-k_{1}}\right)+x_{R_{0}^{\prime}}^{\prime}\left(1-e^{-k_{2} t}\right)+x_{P_{0}}^{\prime}
$$

\section{Calculation of Rate Constants and Activation Parameters}

Data fitting was accomplished using numerical integration and nonlinear least-squares with the default simplex algorithm implemented in the Wolfram Mathematica 10.3. In the following section, we present results from the $2 \mathrm{CR}$ along with $A E$ model.[17] For the $A E$ model, four fitting parameters were used $\left(x_{0}^{\prime}, x_{\infty}^{\prime}, k\right.$, and $\left.m\right)$, while 2CR had five $\left(x_{R_{0}}^{\prime}, x_{R_{0}^{*}}^{\prime}, x_{P_{0}}^{\prime}, k_{1}\right.$ and $\left.k_{2}\right)$. The activation energies for both models were calculated according to the Arrhenius equation, while Gibbs energy of activation, enthalpy of activation and entropy of activation were calculated from Eyring-Polanyi equation.

\section{RESULTS AND DISCUSSION}

\section{Morphology of the Submicrometer Particles of PNND}

The freshly sublimed polymer was studied by SEM microscopy under different magnifications (Figure 2). The polymeric material has interesting morphology. By going to higher magnifications, (Figure $2 \mathrm{~A}-2 \mathrm{C}$ ) the regularity of the (poly)crystalline structure can be inferred. All of the particles are structured in layers, while some of them are also folded (Fig. 2C). The less folded crystals can be seen in figures with the largest magnification (Figure 2D-2E). In our previous paper the molecular geometries of oligomers (up to octamers) were calculated. The molecule is helical and moderately folded. From the length of the octamer, which is $5.1 \pm 0.1 \mathrm{~nm}$, it is possible to estimate that the number of monomeric units (1,4-dinitrosobenzene molecules) in the largest arc in Fig. $2 \mathrm{C}$ might be around $5 \times 10^{3}$. In the smallest edge of the non-folded crystal in the middle of the Fig. $2 \mathrm{~F}$ this number might be less than $4 \times 10^{2}$. The observable folding which appears in larger crystalline particles is expected from the extent of molecular folding found in PNND by calculation. ${ }^{[6]}$ Such a robust structure is a consequence of regular molecular arrangement and as such could be a suitable starting material for the study of the photochemical and thermochemical reaction mechanisms in the solid state.

\section{Photodissociation and Re-dimerization}

According to previous investigations, UV irradiation can be used to induce the dissociation of the azodioxy bond and formation of nitroso monomers, as evidenced by IR spectra. ${ }^{[6]}$ The azodioxy bonds reform upon warming the samples. This is indicated by the decrease of the monomeric $-\mathrm{N}=\mathrm{O}$ signal $\left(1521 \mathrm{~cm}^{-1}\right)$ and the simultaneous increase of the $E$-ONNO signal $\left(1264 \mathrm{~cm}^{-1}\right)$.<smiles>CCCCCCCCCCCCC</smiles><smiles>O=[N+]([O-])c1ccc([N+](=O)[O-])cc1</smiles>

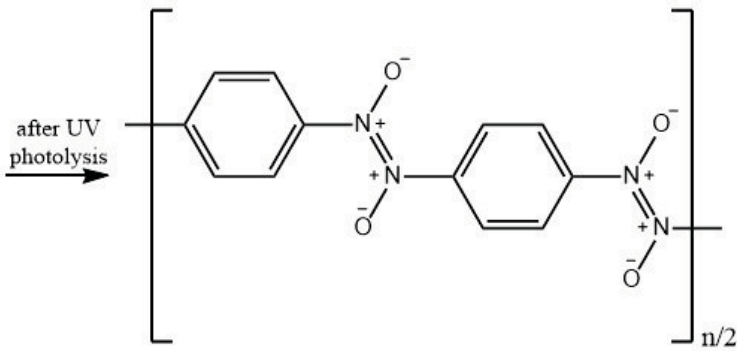

Figure 1. Schematic representation of 1,4-dinitrosobenzene polymerization after deposition onto a cold window and after cryogenic UV photolysis. 

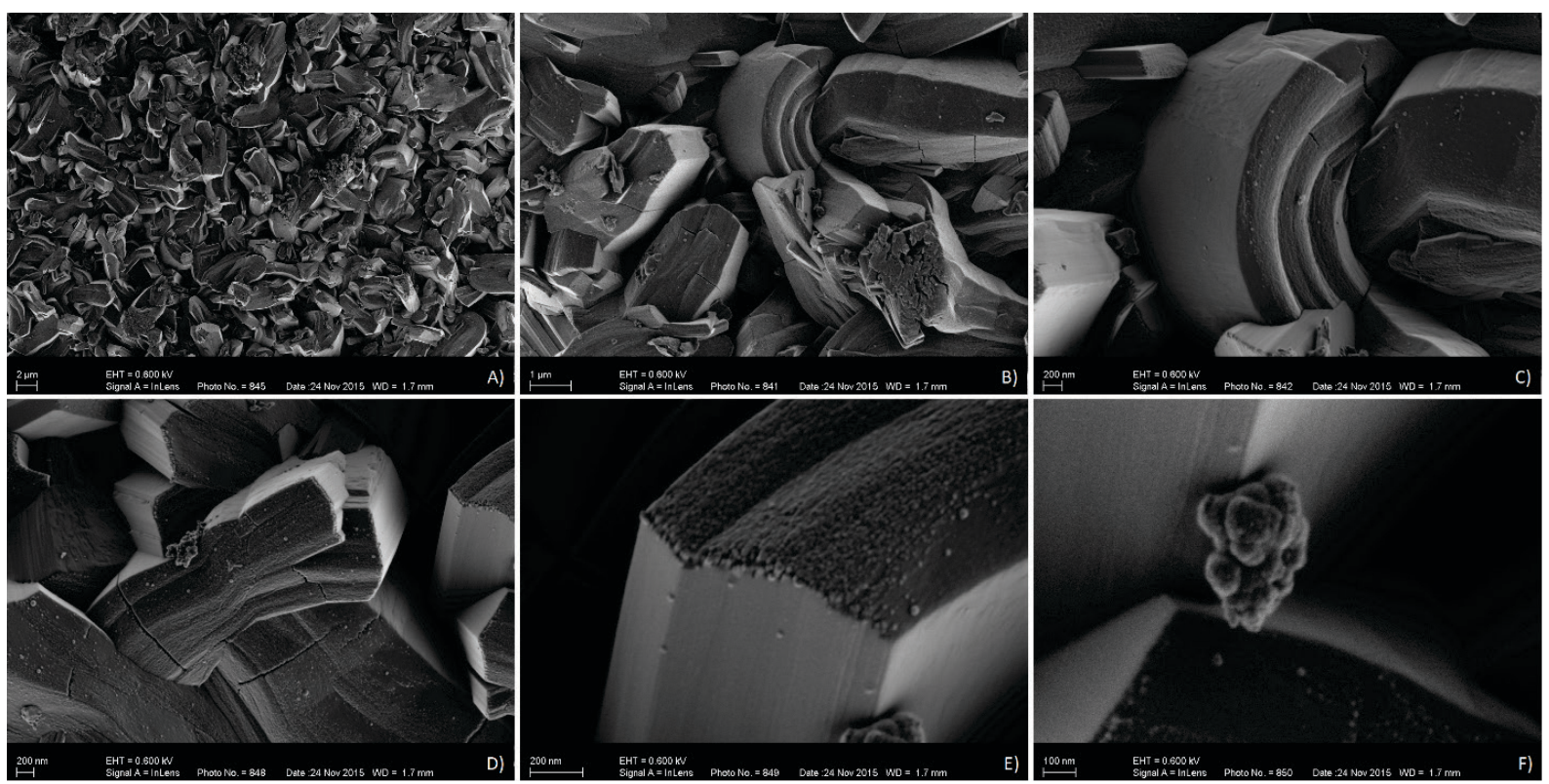

Figure 2. SEM images of the polycrystalline PNND sample at different magnifications.

The optimal temperature range for kinetic measurements of 1,4-dinitrosobenzene polymerization using IR spectroscopy is found to be between 120 and $150 \mathrm{~K}$.

The repolymerization curve features a rapid growth phase, followed by significantly slower roughly linear growth, which resembles some types of aggregative processes. ${ }^{[13]}$ Figure 3 a shows fitted kinetic curves for $2 \mathrm{CR}$ (black) and $\mathrm{AE}$ models (orange), while residuals of the fits are shown in Figure $3 b$. On average, the residual sum of squares of the $A E$ fits are $18 \%$ larger when compared to $2 \mathrm{CR}$ (Table 1). As shown in Figures 3a and $3 b$, the difference is especially pronounced the beginning of the curve, resulting in larger residuals for the AE model.

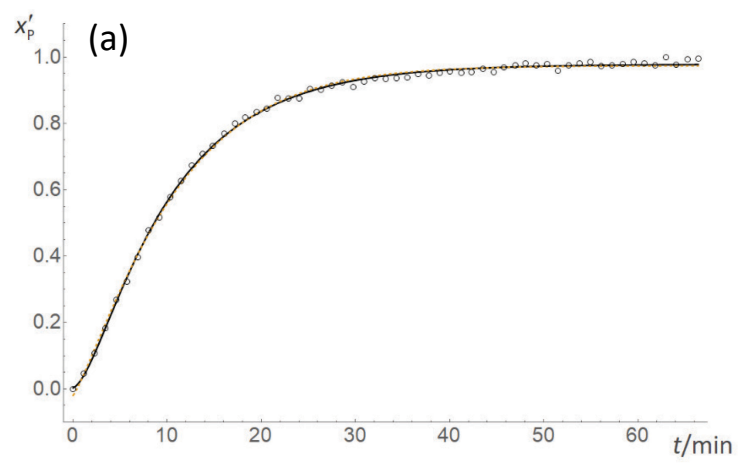

Table 1. Rate constants $\left(k_{2}\right)$ and residual sums of squares (RSS) of the 2CR and AE fitted curves for experiments at different temperatures.

\begin{tabular}{cccccc}
\hline & \multicolumn{3}{c}{$2 \mathrm{CR}$} & \multicolumn{2}{c}{$\mathrm{AE}$} \\
\hline$\#$ & $T / \mathrm{K}$ & $10^{2} \mathrm{k} / \mathrm{min}^{-1}$ & $10^{2} R S S$ & $10^{2} \mathrm{k} / \mathrm{min}^{-1}$ & $10^{2} \mathrm{RSS}$ \\
\hline 1 & 120 & 4.09 & 5.33 & 0.815 & 5.52 \\
2 & 130 & 17.3 & 0.229 & 3.21 & 0.237 \\
3 & 130 & 14.4 & 1.35 & 2.15 & 1.44 \\
4 & 140 & 33.3 & 0.174 & 6.49 & 0.263 \\
5 & 140 & 49.1 & 0.488 & 8.92 & 0.770 \\
6 & 150 & 88.2 & 0.628 & 23.6 & 0.736 \\
\hline
\end{tabular}

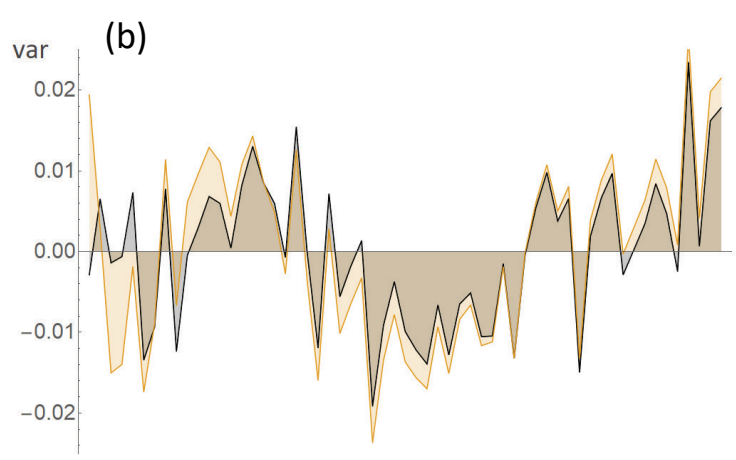

Figure 3. (a) 2CR (black) and AE (orange) fitted curves for repolymerization reaction at $140 \mathrm{~K}$ of the photolyzed sample; (b) $2 \mathrm{CR}$ (black) and $\mathrm{AE}$ (orange) residuals of the fitted curves for repolymerization reaction at $140 \mathrm{~K}$ of the photolyzed sample. 
For the AE model the Arrhenius and Eyring plots are easily obtained, as there is only a single rate constant. However, in case of the $2 \mathrm{CR}$ model there are two rate constants, $k_{1}$ and $k_{2}$. In Figure 4 , it is shown that the slope of the Arrhenius plot for the $2 \mathrm{CR} k_{2}$ constant (black) is very similar to the one obtained for the Avrami $k$ constant. Moreover, activation enthalpies and entropies calculated from the Eyring-Polanyi equation are independent of the used model. These results suggest that the $k_{2}$ constant of the $2 \mathrm{CR}$ model corresponds to the $\mathrm{AE} k$ constant, giving similar activation parameters, as shown in Table 2.

Important contributions of the $2 \mathrm{CR}$ model are the time plots of the reactant, activated reactant and product. As shown in Figure 5, some of the activated reactant is already present at the beginning of the measurement. This might be due to the imperfections in the crystal lattice of the monomer, although a possible facile interconversion between the reactant and the activated reactant cannot be ruled out. In this way, the activated reactant could be an arrangement of molecules similar to the Avrami germ

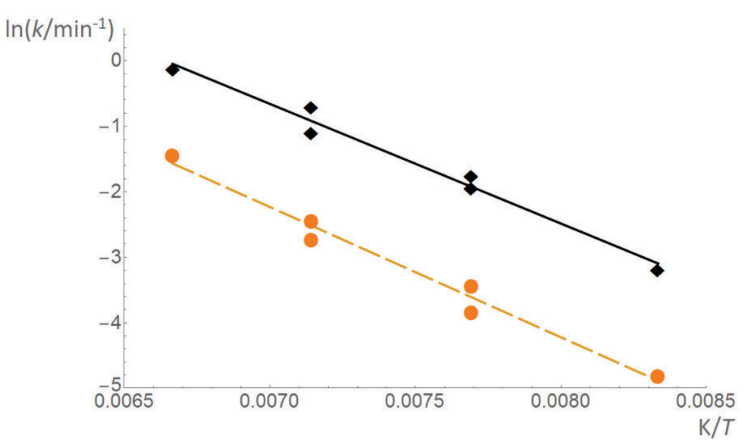

Figure 4. Arrhenius plots for the 1,4-dinitrosobenzene polymerization analyzed by $2 \mathrm{CR}$ model (black) and AE model (orange). The 5 rate constants were obtained from a total of 422 data points.

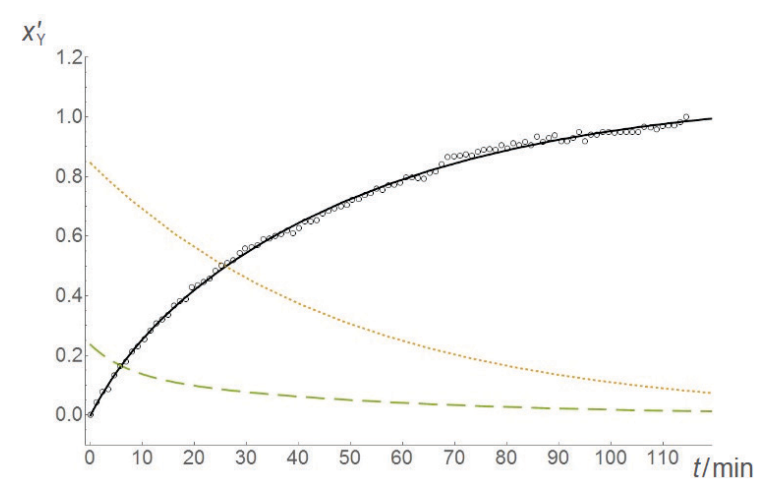

Figure 5. Fitted kinetic curves for reactant, R (orange), activated reactant, $\mathrm{R}^{*}$ (green) and product, $\mathrm{P}$ (black) for the polymerization measurement at $130 \mathrm{~K}$ (entry 3 in Table 1 ) after cryogenic UV photolysis. nuclei. ${ }^{[8-10]}$ These results, along with the Arrhenius plots, suggest that the formation of the activated reactant (by $k_{1}$ ) could correspond to a nucleation-type process, while the $k_{2}$ constant might characterize the chemical reaction.

\section{Polymerization (Dimerization) of the in vacuo Deposited Monomers}

The IR spectra of the deposited samples, when compared to the photolyzed ones, show that different processes take place upon warming. In the deposited sample three parallel processes, displayed in Figures 6 and 7, can be noticed:

At $130 \mathrm{~K}$ the nitroso $\rightarrow$ Z-azodioxy (orange) conversion is dominant, but a relatively slow, parallel nitroso $\rightarrow E$-azodioxy (green) reaction also occurs. However, by warming the sample to $150 \mathrm{~K}$, a very fast, seemingly irreversible formation of the $E$-azodioxy compound takes place at the expense of the Z-isomer (blue). Because these three processes cannot be

Table 2. The calculated activation parameters for the AE and 2CR models.

\begin{tabular}{cccc}
\hline & $E_{a} / \mathrm{kJ} \mathrm{mol}^{-1}$ & $\Delta H^{\ddagger} / \mathrm{kJ} \mathrm{mol}^{-1}$ & $\Delta S^{\ddagger} / \mathrm{J} \mathrm{K}^{-1} \mathrm{~mol}^{-1}$ \\
\hline $\mathrm{AE}$ & $16.6 \pm 1.3$ & $15.5 \pm 1.3$ & $-183.0 \pm 5.1$ \\
$2 \mathrm{CR}\left(k_{2}\right)$ & $15.2 \pm 1.1$ & $14.1 \pm 1.1$ & $-179.7 \pm 9.6$ \\
\hline
\end{tabular}

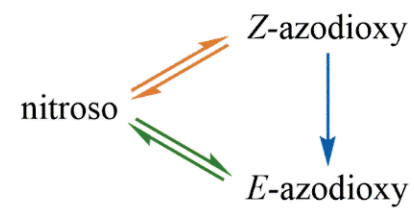

Figure 6. The qualitatively observed reactions in the deposited monomers.

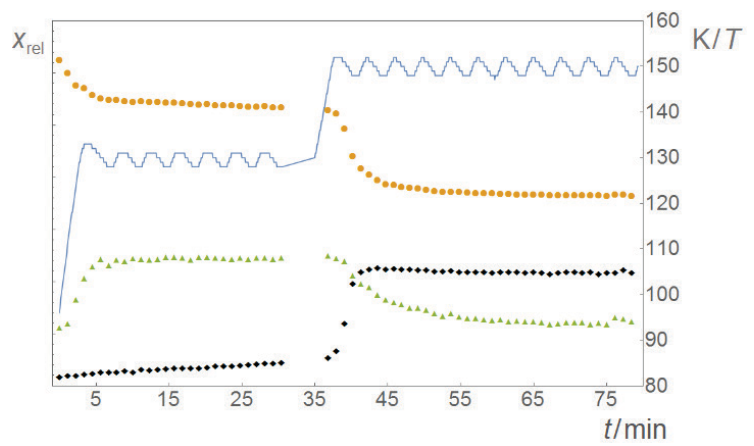

Figure 7. Kinetic profiles for the repolymerization reaction of the deposited sample at 130 and $150 \mathrm{~K}$. Intensities of $-\mathrm{N}=\mathrm{O}, \mathrm{E}$-ONNO and Z-ONNO stretching vibrations are shown in orange, black, and green, respectively. Temperature is indicated by the blue line. 
decoupled, individual rate constants and activation parameters cannot be unambiguously determined in this temperature range.

At higher temperatures the repolymerization kinetics becomes more straightforward, following a quasi-exponential curve. Figure $8 \mathrm{a}$ and $8 \mathrm{~b}$ show the kinetic curve for the repolymerization at $210 \mathrm{~K}$ following cryogenic deposition, analyzed by both $2 \mathrm{CR}$ and $\mathrm{AE}$ models.

The obtained $k_{2}$ and $k$ rate constants for the repolymerization at $210 \mathrm{~K}$ of the deposited sample are $1.01 \mathrm{~min}^{-1}$ and $0.138 \mathrm{~min}^{-1}$, respectively. These rate constants are, on average, 55 times lower than the ones of the photolyzed samples calculated from extrapolation to $210 \mathrm{~K}$. This could indicate that the monomers in samples prepared by photolysis adopt a more favorable topochemistry for chemical reaction than the monomers obtained by deposition.

\section{CONCLUSIONS}

Time-resolved absorbances for the nitroso $\rightarrow$ azodioxy conversions of the photolyzed and deposited PNND samples were measured by IR spectroscopy. The rate

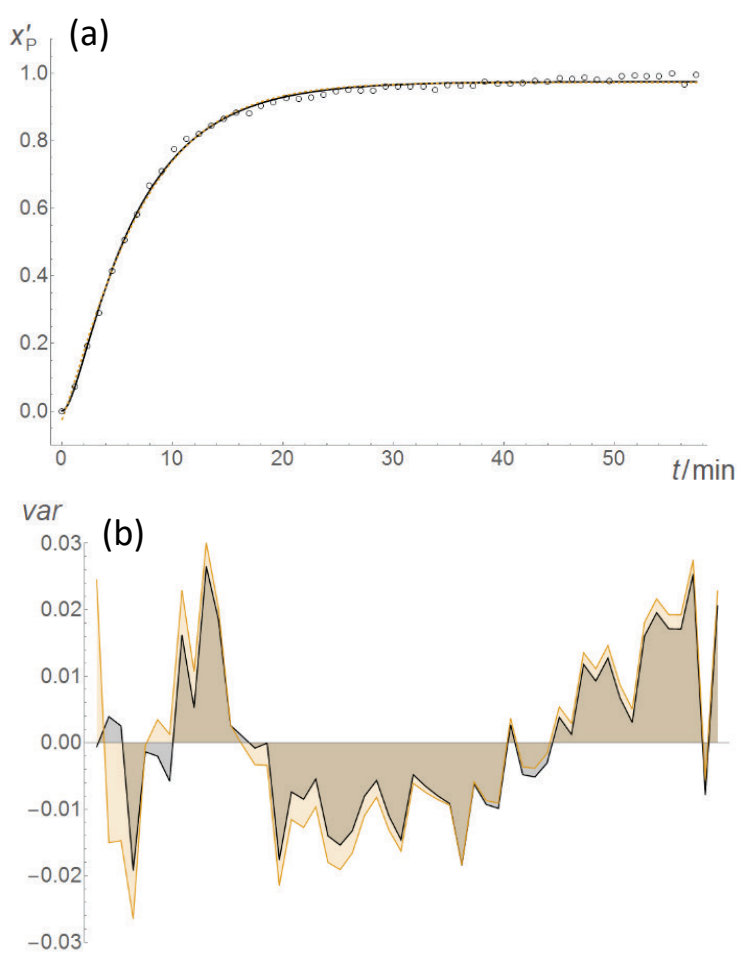

Figure 8. (a) 2CR (black) and AE (orange) fitted curves for repolymerization reaction at $210 \mathrm{~K}$ of the deposited sample; (b) $2 \mathrm{CR}$ (black) and $\mathrm{AE}$ (orange) residuals of the fitted curves for repolymerization reaction at $210 \mathrm{~K}$ of the deposited sample. constants were obtained by curve fitting of the experimental data by both the two-step consecutive reaction ( $2 \mathrm{CR}$ ) and Avrami-Erofeev (AE) models, and the corresponding activation parameters were calculated. Both obtained activation parameters were very similar, at about $15 \mathrm{~kJ} \mathrm{~mol}^{-1}$, suggesting that the $2 \mathrm{CR} k_{2}$ gives similar mechanistic information as the $\mathrm{AE} k$ constant. Such a low $E_{a}$ value could indicate that the monomer units remain close after photolysis, which is in accord with previous findings (specifically, the $\mathrm{N}-\mathrm{N}$ distances in 4-bromonitrosobenzene crystals after photolysis were found to be only $2.3 \AA$ ).${ }^{[1]}$ The overall results, both concerning fits of the individual kinetic curves and the Arrhenius plots, indicate that reaction-order based models, such as $2 \mathrm{CR}$, can be used alongside the $\mathrm{AE}$ model to provide insights about the chemical reactions in the solid state.

The rate constants for the reaction considering the deposited samples proved troublesome to measure precisely because of the overlap of the various processes happening in the temperature range in which reaction proceeds at sufficient rate. Specifically, three coupled processes were detected: the formation of $E$ - and Zazodioxy compounds from monomers, and their interconversion. When compared to the extrapolated rates of the photolyzed samples, the repolymerization reactions of the deposited samples were significantly (by a factor of $\approx 50$ ) slower.

SEM images show that PNND forms semi-regular moieties of the size of up to few $\mathrm{mm}$. From comparing calculated length of polymeric chains from our previous work with the size obtained from SEM measurements, it can be concluded that PNND forms polymers of up to $5 \times 10^{3}$ units long.

Acknowledgment. We thank Professor Zlatko Mihalić for the suggestion to add the 2 CR model and fruitful discussion. We also appreciate precious help of Andre Botha from Laboratory for Microscopy and Microanalysis at University of Pretoria for very kind opportunity to use their equipment. This work was funded by the Croatian Science Foundation, grant no. 7444, project ORGMOL.

\section{REFERENCES}

[1] I. Halasz, E. Meštrović, H. Čičak, Z. Mihalić, H. Vančik, J. Org. Chem. 2005, 70, 8461.

[2] H. Vančik, V. Šimunić-Mežnarić, E. Meštrović, I. Halasz, J. Org. Chem. 2004, 69, 4829.

[3] K. Varga, J. Volarić, H. Vančik, CrystEngComm 2015, 17, 1434.

[4] H. Vančik, Aromatic C-nitroso compounds; Springer, Heidelberg, New York, London, 2001.

[5] D. Beaudoin, J. D. Wuest, Chem. Rev. 2016, 116, 258. 
[6] P. Bibulić, I. Rončević, K. Varga, Z. Mihalić, H. Vančik, J. Mol. Struc. 2016, 1104, 85.

[7] N. P. Hacker, Macromolecules 1993, 26, 5937.

[8] M. Avrami, J. Chem. Phys. 1939, 7, 1103.

[9] M. Avrami, J. Chem. Phys. 1940, 8, 212.

[10] M. Avrami, J. Chem. Phys. 1941, 9, 177

[11] Y. S. Khishchenko, M. A. Makarov, G. A. Gareev, N. A. Cherkashina, G. S. Koptina, Zh. Prikl. Khim. 1969 42, 2384.

[12] A. Khawam, D. R. Flanagan, J. Phys. Chem. B 2006, 110, 17315.
[13] F. Wang, V. N. Richards, S. P. Shields, W. E. Buhro, Chem. Mater. 2014, 26, 5.

[14] M. Olszynski, J. Prywer, A. Torzewska, Cryst. Growth Des. 2015, 15, 3307.

[15] D. T. Moore, H. Sai, K. W. Tan, D.-M. Smilgies, W. Zhang, H. J. Snaith, U. Wiesner, L. A. Estroff, J. Am. Chem. Soc. 2015, 137, 2350.

[16] Y. Pang, D. Sun, Q. Gu, K.-C. Chou, X. Wang, Q. Li, Cryst. Growth Des. 2016, 16, 2404.

[17] Wolfram Research, Inc., Mathematica, Version 10.3, Champaign, IL, 2016. 